\title{
Wireless power transfer using multiple-transmitters for high stability for position
}

\author{
Kazuma Onishi, Kazuya Yamaguchi, Kenichi Iida \\ Department of Control Engineering, National Institute of Technology, Nara College, Japan
}

\begin{tabular}{l} 
Article Info \\
\hline Article history: \\
Received May 24, 2019 \\
Revised Nov 5, 2019 \\
Accepted Nov 24, 2019 \\
\hline
\end{tabular}

\section{Keywords:}

Axial displacement

Multiple transmitter

Wireless power transfer

\begin{abstract}
The aim of this study is to optimize the system in the wireless power supply using multiple transmitters by algebraically analyzing the effects of both a circuit parameter and an axis displacement, etc. In addition to these analyses, the other aim is designing, producing and evaluating wireless charger with high stability for position. In the proposed method, we analyzed the situation in which three transmitters are used as power sources. It is turned out that the optimum arrangement of three transmitters is equilateral triangular from Biot-Savart law and circuit equation. In the experiment, transmitted power is measured when the receiver is moved on the vertical plane in regard to central axis of coils. It is confirmed that $4 \sim 4.5 \mathrm{~mW}$ is transmitted at the face-to-face of transmitters and $2.4 \mathrm{~mW}$ is transmitted at the center of transmitters.
\end{abstract}

Copyright $(5) 2020$ Institute of Advanced Engineering and Science. All rights reserved.

\section{Corresponding Author:}

Kazuya Yamaguchi, Department of Control Engineering, National Institute of Technology, Nara College, 22 Yata-cho, Yamatokoriyama, Nara, Japan.

Email: k-yamaguchi@ctrl.nara-k.ac.jp

\section{INTRODUCTION}

1Wireless power transfer (WPT) has been studied in various fields such as industry $[1,2]$, manufacturing [3], medicine [4, 5], and information [6, 7]. The basic principle has been proposed in the 19th century, and since the MIT report in 2007 [8], it has much attracted many researchers. Recently, many papers about WPT have been published, and various products have been examining and commercializing. For example, studies such as examination of design of coils [9-12] and comparison of efficiency by change of applied voltage waveform $[13,14]$, are investigating. As products, WPT system is applied for power supply on various fields, for instance mobile devices and IoT [15], vehicles traveling on the road [16-19], and micro implant devices [20-22]. As mentioned above, researches finding ideal conditions are actively conducted to improve the performance of WPT [23, 24].

On the other hand, analysis of the situations which degrade performance are not viewed as important, for example leakage flux and misalignment between transmitters and receivers $[25,26]$. As a result, there are some restrictions with relation to design of circuits. On the other hand, considering flexibility of the receiver position, the size of the receiving coil depends on the receiving device, and the loss due to leakage magnetic flux etc. occurs remarkably when transmitting coil is too large. Therefore, it is assumed that it is more advantageous to operate WPT circuit by using a few coils than to handle a single large coil in some situations.

This paper aims realization of stable power transmission regardless of the position of the receiving coil with using multiple transmitters. In order to utilize multiple transmitters, the electromagnetic interaction between transmitters and receiver must be considered. A calculation method is considered and a mathematical formula based on electromagnetism and circuit theory is expressed so that the user could arbitrarily set parameters such as the number and position of transmitters to improve the versatility. Finally, the experimental verification is performed using the actually designed electric circuit, and it is compared with the theoretical result. 


\section{CALCULATION OF POWER}

2.1. Calculation of mutual inductance occurred between two coils arranged in three-dimensional space

Analysis is performed on the case where two coils are arranged in a three-dimensional space in Figure 1. The coordinate of the transmitting coil is $(0,0,0)$, and the coordinate of the receiving coil is $\left(d \_x, d \_y, h\right)$. At this time, d_x and d_y represent an off-axis distance from z-axis, and h represents a facing distance between the coils.

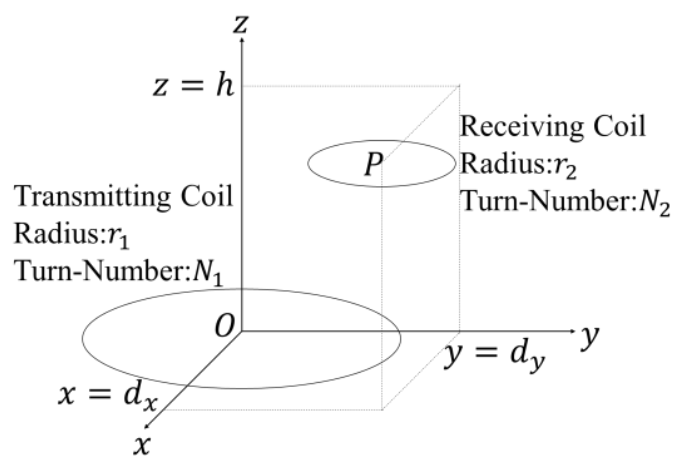

Figure 1. Two coils arranged in three-dimensional space

From the above, the mutual inductance between the coils is obtained according to the Biot-Savart law as follows:

$$
\begin{aligned}
& M \simeq \frac{\mu_{0} S_{1} S_{2}}{2 \pi\left(r_{1}^{2}+d_{x}^{2}+d_{y}^{2}+h^{2}\right)^{\frac{3}{2}}}[\mathrm{H}] \\
& S_{1}=\pi r_{1}^{2} N_{1}, S_{2}=\pi r_{2}^{2} N_{2}
\end{aligned}
$$

\subsection{Power calculation of wireless power transfer circuits using three transmitting circuits}

A wireless power transfer circuit for charging a battery is designed as follows in Figure 2. The left side is a group of transmitting circuits, and the right side is a receiving circuit which contains which battery device. $u$ is the voltage of power supply, $R_{1}, R_{2}, R_{3}, R_{0}, C_{1}, C_{2}, C_{3}, C_{0}$ are parasitic components, $L_{1}, L_{2}, L_{3}, L_{0}$ are the self inductances, $M_{10}, M_{20}, M_{30}$ are mutual inductances between transmitting circuits and receiving circuit, $M_{12}, M_{13}, M_{23}$ are mutual inductances between both transmitting circuits and $R_{L}$ is the resistance of device. From Figure 2, a circuit equation is obtained as (2).

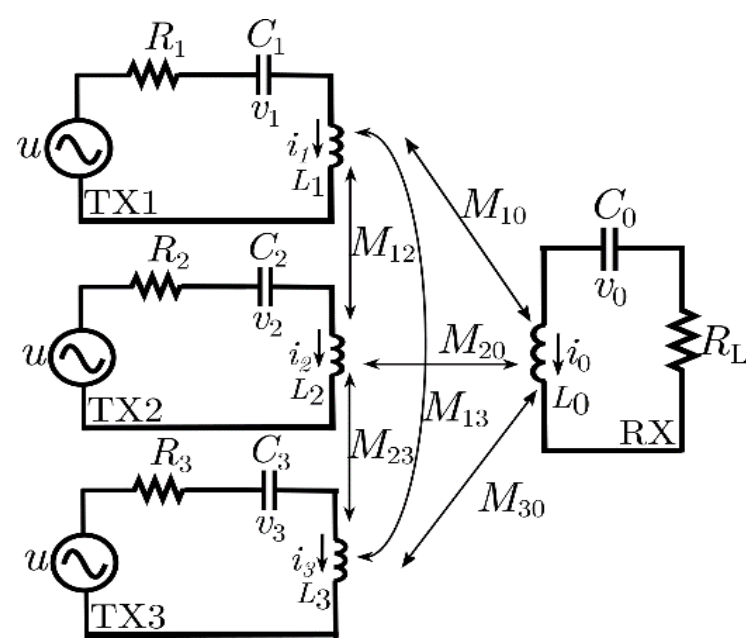

Figure 2. Wireless power transfer circuits using three transmitting circuits 


$$
\begin{aligned}
& {\left[\begin{array}{l}
u \\
u \\
u \\
0
\end{array}\right]=\left[\begin{array}{cccc}
Z_{1} & j \omega M_{12} & j \omega M_{13} & j \omega M_{10} \\
j \omega M_{12} & Z_{2} & j \omega M_{23} & j \omega M_{20} \\
j \omega M_{13} & j \omega M_{23} & Z_{3} & j \omega M_{30} \\
j \omega M_{10} & j \omega M_{20} & j \omega M_{30} & Z_{0}
\end{array}\right]\left[\begin{array}{l}
i_{1} \\
i_{2} \\
i_{3} \\
i_{0}
\end{array}\right]} \\
& Z_{n}=R_{n}+\left(j \omega C_{n}\right)^{-1}+j \omega L_{n}[\Omega], n=0,1,2,3
\end{aligned}
$$

The power at the load $P_{\mathrm{L}}$ can be obtained by solving the current $i_{o}$ of receiving circuit from the above simultaneous equations and substituting it into the following equation:

$$
P_{\text {load }}=R_{\mathrm{L}} i_{0}^{2}[\mathrm{~W}]
$$

\section{EXPERIMENTAL VERIFICATION}

\subsection{Condition of experiment for wireless power transfer by using three transmitting circuits}

The experimental circuit is shown in Figure 3. The values of the circuit elements and the setting of the coordinate conditions are shown in Table 1 and Table 2 respectively.

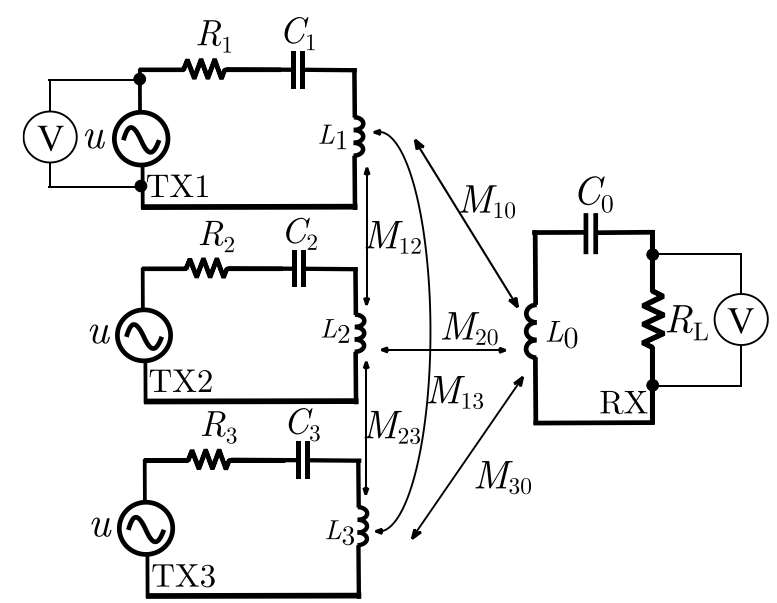

Figure 3. Experimental circuit

Table 1. Values of circuit elements

\begin{tabular}{llllllll}
\hline \multicolumn{7}{c}{ Values of circuit elements } \\
\hline$R_{1}$ & $46.5 \Omega$ & $R_{2}$ & $46.3 \Omega$ & $R_{3}$ & $46.7 \Omega$ & $R_{0}$ & $46.8 \Omega$ \\
$C_{1}$ & $367 \mathrm{pF}$ & $C_{2}$ & $374 \mathrm{pF}$ & $C_{3}$ & $360 \mathrm{pF}$ & $C_{0}$ & $372 \mathrm{pF}$ \\
$L_{1}$ & $24.8 \mu \mathrm{H}$ & $L_{2}$ & $24.8 \mu \mathrm{H}$ & $L_{3}$ & $24.9 \mu \mathrm{H}$ & $L_{0}$ & $24.9 \mu \mathrm{H}$ \\
\hline
\end{tabular}

Table 2. The coordinate conditions

\begin{tabular}{ll}
\hline Parameters & Values [mm] \\
\hline Facing distance between coils $h$ & 15 or 35 \\
Center coordinates of transmitting coils & $(-25-25, h),(25,-25, h),(0,25, h)$ \\
Moving range in the $x$-axis direction & $-50 \sim 50$ \\
Moving range in the $y$-axis direction & $-50 \sim 50$ \\
Moving width in the $x$-axis direction & 5 \\
Moving width in the $y$-axis direction & 10 \\
\hline
\end{tabular}

\subsection{Variation of load power versus receiver coil coordinates}

The variation of load power is investigated by changing the coordinates of the receiving coil with respect to the transmitting coil and the facing distance between the transmitting coil and the receiving coil. The calculated power is shown in Figures 4 and 5, and the experimental power are shown in Figures 6 and 7 , respectively. 


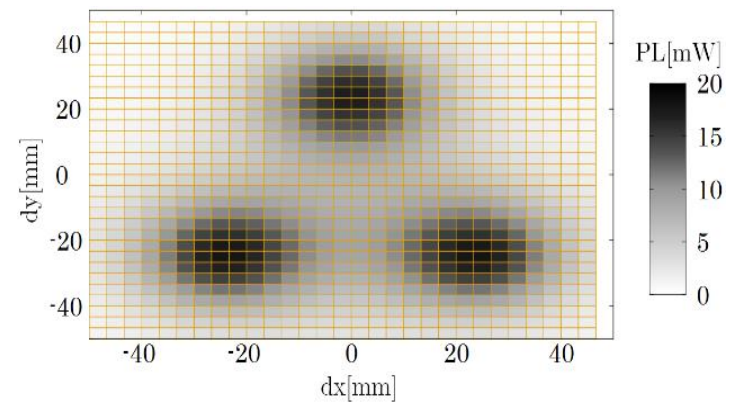

Figure 4. Calculated power where $h=15 \mathrm{~mm}$

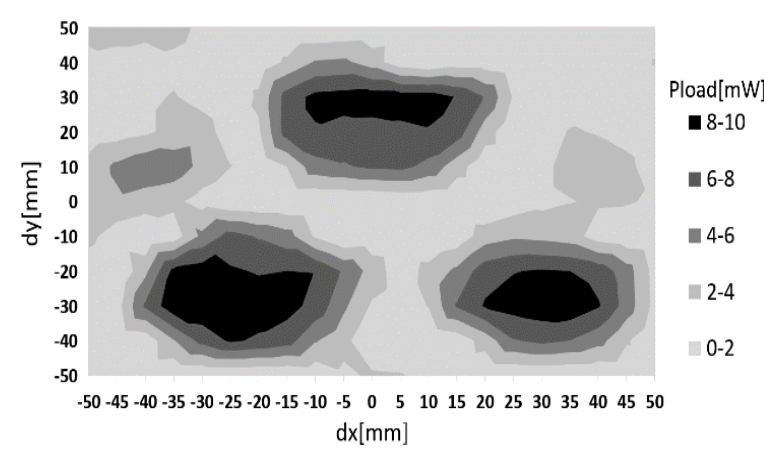

Figure 6. Experimental power where $h=15 \mathrm{~mm}$

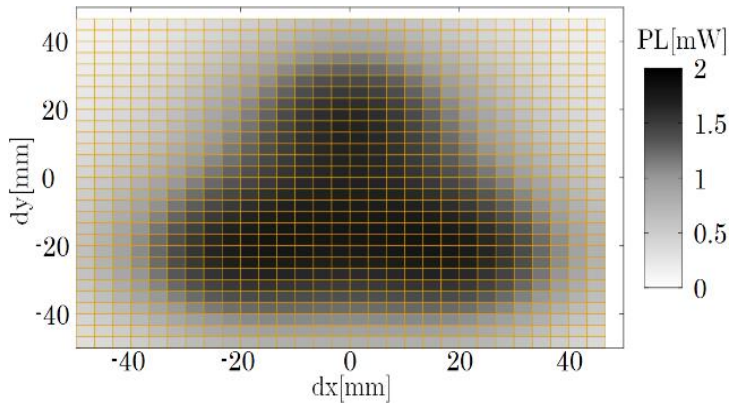

Figure 5. Calculated power where $h=35 \mathrm{~mm}$

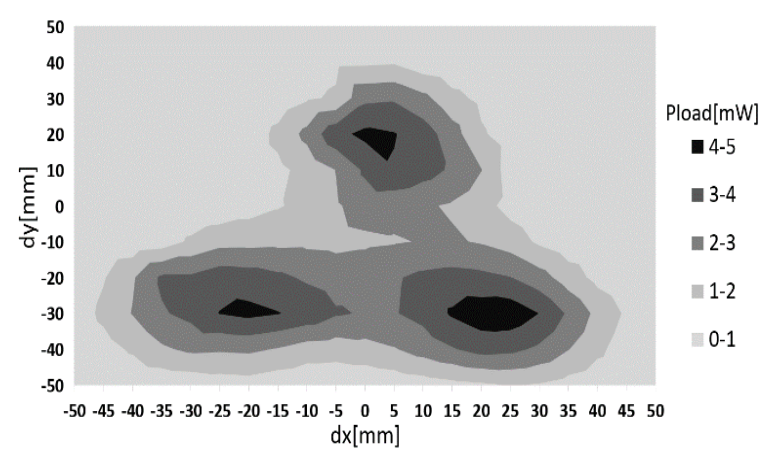

Figure 7. Experimental power where $h=35 \mathrm{~mm}$

\subsection{Discussion}

The comparison of the maximum power and the stability region is shown in Table 3 . Then, the stability region is a range in which the output power is $80 \%$ or more of the maximum power. As the facing distance of the coils increases, the maximum power decreases significantly while the stability region expands. Therefore, it is important to consider the trade-off between power and region in the situation. The defect in the approximation of calculation and experimental circuit are considered as the cause of error.

Table 3. Maximum power and Stability region

\begin{tabular}{ccccc}
\hline & \multicolumn{2}{c}{ Maximum power [mW] } & \multicolumn{3}{c}{ Stability region [\%] } \\
& $h=15 \mathrm{~mm}$ & $h=35 \mathrm{~mm}$ & $h=15 \mathrm{~mm}$ & $h=35 \mathrm{~mm}$ \\
\hline Calculated & 17.82 & 1.585 & 9.62 & 38.4 \\
Experiment & 9.55 & 4.65 & 12.6 & 4.8 \\
\hline
\end{tabular}

\section{CONCLUSION}

This study tried to realize stable wireless power transfer regardless of the arrangement of receiving coil by using multiple transmitters in the circuit. Mutual inductance was derived by using geometrical equation and the power was obtained from the circuit equation considering interactions. Moreover, experiments were performed to prove the validity of the calculation and investigate errors and their causes. As a result, while it was possible to transmit power less than that of expectation, some similarities were found in the trends. In the future, it is necessary to consider the increase in transmission power, the improvement in efficiency, and the effects caused by the increase in coils.

\section{REFERENCES}

[1] Giuseppe Faraci, Angelo Raciti, Santi Agatino Rizzo, Giovanni Schembra, "Green wireless power transfer system for a drone fleet managed by reinforcement learning in smart industry," Applied Energy, Available online 29 November 2019, 114204, pp.1-12, 2019.

[2] Hans De Clercq, Robert Puers, "Contactless energy transfer at the bedside featuring an online power optimization strategy," Sensors and Actuators A 217 (2014), pp.160-167, 2014. 
[3] Massimiliano Amato, Francesco Dalena, Cristina Coviello, Massimo De Vittorio, Simona Petroni, "Modeling, fabrication and characterization of micro-coils as magnetic inductors for wireless power transfer," Microelectronic Engineering 111 (2013), pp.143-148, 2013.

[4] Ada S. Y. Poon, Stephen O’Driscoll, Teresa H. Meng, "Optimal Frequency for Wireless Power Transmission Into Dispersive Tissue," IEEE Transactions on Antennas and Propagation, Vol. 58, No. 5, May 2010, pp.1739-1570, 2010.

[5] Haijun Gu, Zhijun Li, Liheng Wang, Zhuang Ling, "Resource allocation for wireless information and power transfer based on WBAN," Physical Communication 37 (2019) 100865, pp.1-8, 2019.

[6] Zhenyu Na, Yuyao Wang, Xiaotong Li, Junjuan Xia, Xin Liu, Mudi Xiong, Weidang Lu, "Subcarrier allocation based Simultaneous Wireless Information and Power Transfer algorithm in 5G cooperative OFDM communication systems," Physical Communication 29 (2018), pp.164-170, 2018.

[7] Dinh-Thuan Do, Minh-Sang Van Nguyen, "Device-to-device transmission modes in NOMA network with and without Wireless Power Transfer," Computer Communications 139(2019), pp.67-77, 2019.

[8] A. Kurs, A. Karalis, R. Moffatt, J. D. Joannopoulos, P. Fisher, and M. Solja ci'c, "Wireless Power Transfer via Strongly Coupled Magnetic Resonances," Science, vol. 317, pp. 83-86, 2007.

[9] S. Chatterjee, A. Iyer, C. Bharatiraja, I. Vaghasia, and V. Rajesh, "Design Optimisation for an Efficient Wireless Power Transfer System for Electric Vehicles," Energy Procedia, vol. 117, pp. 1015-1023, 2017.

[10] C.A. Tucker, K. Warwick, W. Holderbaum, "A contribution to the wireless transmission of power," Electrical Power and Energy Systems 47 (2013), pp.235-242, 2013.

[11] Xiufang Wang, Xinyi Nie, Yilang Liang, Falong Lu, Zhongming Yan, Yu Wang, "Analysis and experimental study of wireless power transfer with HTS coil and copper coil as the intermediate resonators system," Physica C: Superconductivity and its applications 532 (2017), pp.6-12, 2017.

[12] Alanson P. Sample,David, T. Meyer, Joshua R. Smith, "Analysis, Experimental Results, and Range Adaptation of Magnetically Coupled Resonators for Wireless Power Transfer," IEEE Transactions on Industrial Electronics, Vol.58, No.2, Feb.2011, pp.544-554, 2011.

[13] Kazuya Yamaguchi, Takuya Hirata, Ichijo Hodaka, "High Power Wireless Power Transfer Driven by Square Wave Inputs," Proceedings of the 9th International Conference on ICGEC '15, pp. 341-346, 2015.

[14] Shitong Mao, Hao Wanga,b, Chunbo Zhu, Zhi-Hong Mao, Mingui Sun, "Simultaneous wireless power transfer and data communication using synchronous pulse-controlled load modulation," Measurement 109 (2017), pp.316-325, 2017.

[15] L. Han, and L. Li, "Integrated wireless communications and wireless power transfer: An overview," Physical Communication, vol. 25, part 2, pp. 555-563, 2017.

[16] Katsuhiro Hata, Kensuke Hanajiri, Takehiro Imura, Hiroshi Fujimoto, Yoichi Hori, Motoki Sato, Daisuke Gunji, "Design and Implementation of Sensorless Vehicle Detection System for In-motion Wireless Power Transfer," The 31st International Electric Vehicle Symposium \& Exhibition and International Electric Vehicle Technology Conference 2018 (EVS31\&EVTeC2018), pp.1-7, 2018.

[17] Kafeel Ahmed Kalwar, Muhammad Aamir, Saad Mekhilef, "Inductively coupled power transfer (ICPT) for electric vehicle charging- A review," Renewable and Sustainable Energy Reviews 47 (2015), pp.462-475, 2015.

[18] Bo Zhang, Richard B. Carlson, John G. Smart, Eric J. Dufek, Boryann Liaw, "Challenges of future high power wireless power transfer for light-duty electric vehicles -technology and risk management," eTransportation 2 (2019) 100012, pp.1-13, 2019.

[19] John S. Ho, Alexander J. Yeh, Evgenios Neofytou, Sanghoek Kim, Yuji Tanabe, Bhagat Patlolla, Ramin E. Beygui, and Ada S. Y. Poon, "Wireless power transfer to deep-tissue microimplants, "PNAS June 3, pp. 7974-7979, 2014.

[20] Md. Rubel Basar, Mohd Yazed Ahmad, Jongman Cho, Fatimah Ibrahim, "An improved resonant wireless power transfer system with optimum coil configuration for capsule endoscopy," Sensors and Actuators A 249 (2016), pp.207-216, 2016.

[21] Anthony N. Laskovski, Mehmet R. Yuce, "Class-E self-oscillation for the transmission of wireless power to implants," Sensors and Actuators A 171 (2011), pp.391-397, 2011.

[22] Zicheng Bi, Tianze Kan, Chunting Chris Mi, Yiming Zhang, Zhengming Zhao, Gregory A. Keoleian, "A review of wireless power transfer for electric vehicles: Prospects to enhance sustainable mobility," Applied Energy 179 (2016), pp.413-425, 2016.

[23] TAN LinLin, HUANG XueLiang, HUANG Hui, ZOU YuWei and LI Hui, "Transfer efficiency optimal control of magnetic resonance coupled system of wireless power transfer based on frequency control," SCIENCE CHINA Technological Sciences June 2011, vol. 54 no.6, pp.1428-1434, 2011.

[24] Giulia Di Capua, Nicola Femia, Giovanni Petrone, Gianpaolo Lisi, Dingkun Du, Rajaram Subramonian, "Power and efficiency analysis of high-frequency Wireless Power Transfer Systems," Electrical Power and Energy Systems 84 (2017), pp.124-134, 2017.

[25] Sousuke NAKAMURA, Ryo KOMA, and Hideki HASHIMOTO, "Efficient Wireless Power Transmission based on Position Sensing using Magnetic Resonance Coupling," SICE Journal of Control, Measurement and System Integration, Vol.5, No.3, pp.153-161, 2012.

[26] Y. P. Su, Xun Liu, S. Y. Ron Hui, "Mutual Inductance Calculation of Movable Planar Coils on Parallel Surfaces," IEEE Transactions on Power Electronics, Vol. 24, No. 4, April 2009, pp.1115-1124, 2009. 